\title{
Pacific
}

Journal of

Mathematics

\section{THE BOUNDARY DISTORTION OF A QUASICONFORMAL} MAPPING

Juha Heinonen and PeKka Koskela

Volume 165 No. 1 


\title{
THE BOUNDARY DISTORTION OF A QUASICONFORMAL MAPPING
}

\author{
Juha Heinonen and PekKa Koskela
}

\begin{abstract}
We investigate how quasiconformal mappings $f: \mathbf{B}^{n} \rightarrow \mathbf{R}^{n}$ distort Hausdorff measures of sets on the boundary $\partial \mathbf{B}^{n}$.
\end{abstract}

1. Introduction. Recent exciting developments in the study of the boundary behavior of conformal mappings, orchestrated by Makarov ([M1], [M2], [M3]), lead to the natural question: what is the boundary distortion of a quasiconformal mapping $f$ of the unit ball $\mathbf{B}^{n}$ into $\mathbf{R}^{n}$ ? Makarov's results assert that for any conformal mapping of the unit disk in the plane there is a set of full measure on the boundary whose image has Hausdorff dimension precisely one. There is no hope for such results for general quasiconformal mappings as shown by wellknown examples. In fact, given $0<\alpha<1$ there is a quasiconformal self-homeomorphism of the unit disk carrying a set of full measure on the circle into a set of Hausdorff dimension $\alpha$ (see [Ro], [T2]). In the other direction, given $1<\alpha<2$ it is not difficult to construct a quasiconformal mapping of the disk onto a Jordan domain such that the image of any boundary set of positive measure has Hausdorff dimension $\alpha$.

In this article we discuss various questions related to the boundary distortion of quasiconformal mappings; in particular, we demonstrate that in spite of the discouraging counterexamples at least some features of the restricted expansion/contraction phenomenon are retained. Naturally, our results lag behind the deep information available in the case of conformal mappings, but we feel that some of the techniques used in this paper are of interest in the higher dimensional quasiconformal theory. It is also our hope that the modest beginning here will inspire future research in this area. Several open questions are listed at the end of the paper.

2. Main results. In this section we describe our main results. The proofs will follow in subsequent sections.

2.1. Notation. We let $B(x, r)$ stand for the open $n$-ball centered at $x$ with radius $r$, and we assume $n \geq 2$. For short, $B_{r}=B(0, r)$ 
and $\mathbf{B}^{n}=B(0,1)$. The boundary, the complement, and the closure of a set $E \subset \mathbf{R}^{n}$ are denoted by $\partial E, C E$, and $\bar{E}$, respectively. We write $\mathscr{H}_{s}$ for the $s$-dimensional Hausdorff measure and usually, for simplicity, we write $\mathscr{H}_{n-1}(E)=|E|$. Also, $\operatorname{dim}_{H} E$ denotes the Hausdorff dimension of a set $E$. We often let $C$ denote a generic positive constant which is not necessarily the same at each occurrence. Moreover, sometimes we write $A \approx B$ if there is $C$ such that $C^{-1} A \leq B \leq C A$.

The conformal $(n)$-modulus of a path family $\Gamma$ will be denoted by mod $\Gamma$. Often $\Gamma=\Delta(E, F ; A)$, the family of all paths joining two sets $E$ and $F$ in $A$. For the basic properties of the modulus, see [V1].

The outer dilatation $K_{O}(f)$ of a homeomorphism $f: D \rightarrow D^{\prime}$ is defined by

$$
K_{O}(f)=\sup \frac{\bmod \Gamma}{\bmod f \Gamma},
$$

where the supremum is over all path families $\Gamma$ in $D$ such that $\bmod f \Gamma \neq 0$. Then the inner dilatation is $K_{I}(f)=K_{O}\left(f^{-1}\right)$ and $f$ is said to be $K$-quasiconformal if

$$
K(f)=\max \left\{K_{O}(f), K_{I}(f)\right\} \leq K<\infty .
$$

We recall the basic relations

$$
K_{O}(f) \leq K_{I}(f)^{n-1}, \quad K_{I}(f) \leq K_{O}(f)^{n-1} .
$$

If $f$ is a quasiconformal mapping of $\mathbf{B}^{n}$ into $\mathbf{R}^{n}$, then an analog of Beurling's theorem ensures that $f$ has angular limits at each point on the boundary $\partial \mathbf{B}^{n}$ except possibly in a set $E_{f}$ of zero $n$-capacity. In particular, $E_{f}$ has Hausdorff dimension zero (see, for instance, [N, Corollary 7.14], [Vu, Chapter IV]). In the following we understand that $f$ is defined on $\partial \mathbf{B}^{n}$ via its radial extension; it is immaterial what the value of $f$ is in the exceptional set $E_{f}$.

The standard reference to the basic theory of quasiconformal mappings is [V1].

2.2. Contraction. Our first three results concern the boundary compression of quasiconformal mappings.

THEOREM A. Suppose that $f$ is a quasiconformal mapping of $\mathbf{B}^{n}$ into $\mathbf{R}^{n}$. Then for any set $E \subset \partial \mathbf{B}^{n}$ with positive $\mathscr{H}_{n-1}$-measure, the Hausdorff dimension of $f(E)$ is at least

$$
(n-1)\left(2 K_{O}(f)\right)^{1 /(1-n)} \text {. }
$$


If $n=2$ and $f$ is conformal, we recover the classical Beurling estimate for the support of harmonic measure. In fact, the idea of the proof for Theorem A goes back to Beurling. The lower bound in Theorem $\mathrm{A}$ is undoubtedly not sharp. It is perhaps too optimistic to hope for the bound $(n-1)\left(K_{O}(f)\right)^{1 /(1-n)}$, which would give Makarov's result as a special case, but a reasonable conjecture is $(n-1)\left(2 K_{O}(f)\right)^{1 /(1-n)}+\varepsilon$ for some $\varepsilon=\varepsilon(n, K)>0$. This would correspond to Carleson's result [C] for conformal mappings. Although Carleson's ingenious method morally works for quasiconformal mappings, it is not clear to us how to transfer the final steps in his argument into $n$-space. If we place an additional constraint on the target domain, an improvement to Theorem $\mathrm{A}$ is immediate.

Recall that a domain $D \subset \mathbf{R}^{n}$ is $b$-uniform if each pair of points $x, y \in D$ can be joined by an arc $L$ in $D$ such that

$$
\operatorname{diam} L \leq b|x-y|
$$

and

$$
\min \{\operatorname{diam} L[x, z], \operatorname{diam} L[y, z]\} \leq b \operatorname{dist}(z, \partial D)
$$

whenever $z \in L$ and $L[w, z]$ denotes the subarc of $L$ between $w$ and $z$.

THEOREM B. Suppose that $f$ is a quasiconformal mapping of $\mathbf{B}^{n}$ onto a b-uniform domain. Then for any set $E \subset \partial \mathbf{B}^{n}$ with positive $\mathscr{H}_{n-1}$-measure, the Hausdorff dimension of $f(E)$ is at least

$$
(n-1)\left(2 K_{O}(f)\right)^{1 /(1-n)}+\varepsilon,
$$

where $\varepsilon>0$ depends only on $n, K_{O}(f)$, and $b$.

Theorem $B$ is a consequence of the following result which extends [NP, Theorem 9].

THEOREM $\mathbf{B}^{\prime}$. Suppose that $f$ is a quasiconformal mapping of $\mathbf{B}^{n}$ onto a b-uniform domain $D$. Then $f^{-1}$ is Hölder continuous in $D$ with exponent $\alpha=\left(2 K_{O}(f)\right)^{1 /(1-n)}+\varepsilon$ for some $\varepsilon=\varepsilon\left(n, K_{O}(f), b\right)>$ 0 .

The distortion of general Hausdorff measures is described in the next theorem. We let $D f$ denote the differential of a quasiconformal mapping $f$, which exists almost everywhere in the domain of $f$. 
THEOREM C. Suppose that $f$ is a quasiconformal mapping of $\mathbf{B}^{n}$ into $\mathbf{R}^{n}$ such that $|D f|^{-r} \in L^{1}\left(\mathbf{B}^{n}\right)$ for $r>0$. Then for any set $E \subset \partial \mathbf{B}^{n}$ with positive $\mathscr{H}_{s}$-measure, $0<s \leq n-1$, the Hausdorff dimension of $f(E)$ is at least

$$
\frac{s r}{n-s+r}
$$

Astala and Koskela [AK] have shown that if $f$ is a $K$-quasiconformal mapping of $\mathbf{B}^{n}$, then always $|D f|^{-r} \in L^{1}\left(\mathbf{B}^{n}\right)$ for some $r=$ $r(n, K)>0$. Thus Theorem $\mathrm{C}$ is never vacuous. The exact value of $r(n, K)$ is not known but for $n \geq 3$ it satisfies $r(n, K) \rightarrow \infty$ as $K \rightarrow$ 1 (see [Re, Chapter 4]). For conformal mappings Pommerenke [P1] has obtained the lower bound $r(2,1) \geq 1.39$. Moreover, Carleson and Makarov [CM] have recently established that if $f$ is univalent in the unit disk $\mathbf{B}^{2}$ and $E \subset \partial \mathbf{B}^{2}$ with positive $\mathscr{H}_{s}$-measure, $0<s<1$, then the Hausdorff dimension of $f(E)$ is at least $s /(2-a s)$, where $0<a<2$ is an absolute constant (see also [M2]). By using this result and factorization, we see that

$$
\operatorname{dim}_{H} f(E) \geq \frac{s}{2 K(f)-a s}
$$

whenever $E \subset \partial \mathbf{B}^{2}$ is as above and $f$ is quasiconformal in $\mathbf{B}^{2}$; note that $K(f)=K_{O}(f)=K_{I}(f)$ in the plane.

We shall show in Lemma 5.1 below that if $n \neq 4$ and if $f$ is as in Theorem $\mathrm{C}$, then there is a quasiconformal mapping $g$ of $\mathbf{B}^{n}$ such that $g=f$ on $\partial \mathbf{B}^{n}$ and that $|D g|^{-r} \in L^{1}\left(\mathbf{B}^{n}\right)$ for all

$$
0<r<\frac{1}{\left(2 K_{O}(f)\right)^{1 /(n-1)}-1}
$$

thus for $n \neq 4$ Theorem A follows from Theorem $\mathrm{C}$.

If $f$ is a $K$-quasiconformal self mapping of $\mathbf{R}^{n}$, then it follows from [G1, Theorem 3] that

$$
\frac{\operatorname{dim}_{H} f(E)}{\operatorname{dim}_{H} E} \geq \frac{p(n, K)-n}{p(n, K)-\operatorname{dim}_{H} E}
$$

for any set $E \subset \mathbf{R}^{n}$, where $p(n, K)>n$ is defined to be the supremum of all $p>n$ such that $|D f| \in L_{\text {loc }}^{p}\left(\mathbf{R}^{n}\right)$. Also, if $n=2$ and if $f$ has a $K$-quasiconformal extension to the whole plane, Astala's recent affirmative solution to the area distortion problem [A] guarantees the lower bound $2(1+K)^{-1}$ in Theorem A. We emphasize that in Theorems $\mathrm{A}$ and $\mathrm{C} f$ need not have to have a continuous extension to $\partial \mathbf{B}^{n}$. 
2.3. Expansion. Now we turn our attention to the other aspect of the Makarov theory, namely expansion. Makarov's insight was to show that if the derivative of a conformal mapping $f$ satisfies

$$
\liminf _{r \rightarrow 1}\left|f^{\prime}(r w)\right|=0
$$

for all $w$ in a set $E \subset \partial \mathbf{B}^{2}$, then $f$ cannot expand at almost all points in $E$. Consequently, since $f^{\prime}$ is analytic, Plessner's theorem implies that the expansion occurs in a subset of zero measure on $\partial \mathbf{B}^{2}$. For a quasiconformal mapping $f: D \rightarrow D^{\prime}$ there is, in general, no pointwise defined derivative but the integral average

$$
a_{f}(x)=\exp \left\{\frac{1}{n} f_{B_{x}} \log J_{f}(y) d y\right\}
$$

has proved to be a good substitute; here $B_{x}=B(x, \operatorname{dist}(x, \partial D) / 2)$ (see [AG1], [AG2], [AK], [Ha], [K2]). Take notice that if $f$ is conformal, then $\log J_{f}(x)=2 \log \left|f^{\prime}(x)\right|$ is harmonic, whence $a_{f}(x)=$ $\left|f^{\prime}(x)\right|$.

Pommerenke [P2] simplified and strengthened Makarov's argument in [M1] as regards the expansion. We simplify it a bit further and show that more or less the same proof goes through for quasiconformal mappings. Of course Plessner's theorem is not applicable in this case and we have to be content with the following formulation.

THeOREM D. Suppose that $f$ is a quasiconformal mapping of $\mathbf{B}^{n}$ into $\mathbf{R}^{n}$. Let

$$
E_{\infty}=\left\{w \in \partial \mathbf{B}^{n}: 0<\liminf _{r \rightarrow 1} a_{f}(r w) \leq \limsup _{r \rightarrow 1} a_{f}(r w)=\infty\right\} .
$$

Then

$$
\partial \mathbf{B}^{n}=E_{0} \cup E_{1} \cup E_{\infty} \quad \text { (disjoint union) }
$$

where $\left|E_{0}\right|=0$ and $f\left(E_{1}\right)$ has $\sigma$-finite $\mathscr{H}_{n-1}$-measure. Moreover, if $E \subset E_{1}$, then $|E|=0$ implies $|f(E)|=0$.

Pommerenke's version of Makarov's theorem follows from Theorem D. Indeed, if $f$ is conformal, then $a_{f}(x)=\left|f^{\prime}(x)\right|$ and Plessner's theorem implies $\left|E_{\infty}\right|=0$.

Definition. We say that a quasiconformal mapping $f$ of $\mathbf{B}^{n}$ into $\mathbf{R}^{n}$ has the Makarov property if there is a set $E$ of full measure on the boundary of $\mathbf{B}^{n}$ such that $f(E)$ has $\sigma$-finite $(n-1)$-measure. 
By Theorem D, $f$ has the Makarov property if for almost every $w \in \partial \mathbf{B}^{n}$ either

$$
\liminf _{r \rightarrow 1} a_{f}(r w)=0 \quad \text { or } \quad \limsup _{r \rightarrow 1} a_{f}(r w)<\infty .
$$

As mentioned in the introduction, this need not always be the case. For instance, one can construct a quasiconformal mapping $f$ of $\mathbf{R}^{2}$ that carries the unit circle onto the van Koch snowflake such that

$$
|f(z)-f(w)| \approx|z-w|^{\alpha}, \quad \alpha=\frac{\log 3}{\log 4},
$$

for all $z, w \in \partial \mathbf{B}^{2}$ (see [T1]). It is easy to see that then the image of any set $E \subset \partial \mathbf{B}^{2}$ of positive length has Hausdorff dimension $1 / \alpha>1$. In fact, in this case $\lim _{r \rightarrow 1} a_{f}(r w)=\infty$ for all $w \in \partial \mathbf{B}^{2}$.

Incidentally, we do not know of any examples in dimensions $n \geq 3$ where $E_{\infty}$ has positive $\mathscr{H}_{n-1}$-measure. Väisälä [V6] has constructed a quasiconformal mapping of the unit ball $\mathbf{B}^{n}$ onto a Jordan domain whose boundary has positive volume; it would be interesting to know the behavior of $a_{f}$ for such a mapping. Another interesting open problem is to find conditions (other than $\left|E_{\infty}\right|=0$ ) that would guarantee the Makarov property. In dimension $n=2$ we have the following sufficient condition which is an immediate consequence of a recent work of Fefferman, Kenig, and Pipher [FKP].

THEOREM E. Suppose that $f$ is a quasiconformal mapping of $\mathbf{B}^{2}$ into $\mathbf{R}^{2}$. Let $\mu(z)$ denote the complex dilatation of $f$ for $z \in \mathbf{B}^{2}$. If the function

$$
\frac{|\mu(z)|^{2}}{1-|z|}
$$

is a Carleson measure in $\mathbf{B}^{2}$, then there is a set $E \subset \partial \mathbf{B}^{2}$ such that $|E|=2 \pi$ and $f(E)$ has Hausdorff dimension 1. Moreover, $f$ has the Makarov property.

Recall that a measure $\nu$ in $\mathbf{B}^{2}$ is a Carleson measure if there is a constant $C>0$ such that $\nu\left(B \cap \mathbf{B}^{2}\right) \leq C$ diam $B$ for each disk $B$ centered at a point on $\partial \mathbf{B}^{2}$.

Theorem E tells us that if the dilatation of a quasiconformal mapping becomes small on the boundary in a certain uniform sense, the boundary distortion is not different from that of a conformal mapping. We do not know to what extent Theorem $\mathrm{E}$ is sharp for the class of mappings that become "conformal at the boundary". 
It is clear that a quasiconformal mapping can have the Makarov property without its dilatation tending to zero as we approach the boundary, but it seems difficult to give nontrivial conditions guaranteeing this.

As our final result we state an analog of Theorem $\mathrm{E}$ for $n \geq 3$. Because 1-quasiconformal mappings in space are Möbius transformations, the following theorem comes as no surprise.

THEOREM F. Suppose that $n \geq 3$ and that $f$ is a quasiconformal mapping of $\mathbf{B}^{n}$ into $\mathbf{R}^{n}$ such that

$$
K\left(f \mid\left(\mathbf{B}^{n} \backslash \bar{B}_{r_{j}}\right)\right) \rightarrow 1,
$$

where $r_{j} \uparrow 1$. Then for any set $E \subset \partial \mathbf{B}^{n}$ with Hausdorff dimension $s$, $0<s \leq n-1$, the Hausdorff dimension of $f(E)$ is $s$.

We do not know whether the mapping $f$ in Theorem $\mathrm{F}$ has the Makarov property.

3. Proof of Theorem A. Let $f: \mathbf{B}^{n} \rightarrow D$ be a $K$-quasiconformal mapping; we normalize $f(0)=0$ and $\operatorname{dist}(0, \partial D)=1$. Fix a point $y \in \partial D$ and let $0<r<d / 2$, where

$$
d=d(n, K)=\operatorname{dist}\left(f \bar{B}_{1 / 2}, \partial D\right) .
$$

Then for the path family

$$
\Gamma^{\prime}=\Lambda\left(f \bar{B}_{1 / 2}, \bar{B}(y, r) \cap \partial D ; D\right)
$$

we have the estimate

$$
\bmod \Gamma^{\prime} \leq \frac{\omega_{n-1}}{(\log d / r)^{n-1}}
$$

and so

$$
\bmod \Gamma \leq \frac{K_{O} \omega_{n-1}}{(\log d / r)^{n-1}},
$$

where $\Gamma=f^{-1} \Gamma^{\prime}$ and $K_{O}=K_{O}(f)$. Let $E$ denote an arbitrary compact subset of the set of all boundary points $w \in \partial \mathbf{B}^{n}$ such that the radial limit $\lim _{t \rightarrow 1} f(t w)=f(w)$ exists and satisfies $|f(w)-y| \leq$ $r$. Let

$$
\Gamma_{1}=\left(\bar{B}_{1 / 2}, E ; \mathbf{B}^{n}\right) .
$$

If $w \in E$ and $\gamma \in \Gamma_{1}$, then by Lindelöf's theorem for quasiconformal mappings either $f(\gamma)$ is nonrectifiable or $f$ has the limit $f(w) \in$ 
$\bar{B}(y, r)$ along $\gamma$. Because the modulus of nonrectifiable paths is zero, we conclude that

$$
\bmod \Gamma_{1} \leq \bmod \Gamma .
$$

By reflecting one can show that

$$
\bmod \Gamma_{0} \leq 2 \bmod \Gamma_{1},
$$

where

$$
\Gamma_{0}=(E, \partial B(0,2) ; B(0,2)),
$$

(see e.g. [HM, Lemma 3.8]). Next, if we replace $E$ by a (possibly degenerate) spherical cap $E^{*} \subset \partial \mathbf{B}^{n}$ such that $|E|=\left|E^{*}\right|$, then

$$
\bmod \Gamma_{0}^{*} \leq \bmod \Gamma_{0},
$$

where $\Gamma_{0}^{*}$ is the symmetrized family

$$
\Gamma_{0}^{*}=\left(E^{*}, \partial B(0,2) ; B(0,2)\right)
$$

see [S]. Let

$$
r_{1}=\operatorname{diam} E^{*} \approx\left|E^{*}\right|^{1 /(n-1)} .
$$

A symmetrization argument (see [HM, (3.14)]) combined with the above inequalities (3.1)-(3.4) yield

$$
\begin{aligned}
r_{1} & \leq c_{n} \exp \left\{-\omega_{n-1}^{1 /(n-1)}\left(\bmod \Gamma_{0}^{*}\right)^{1 /(1-n)}\right\} \\
& \leq c_{n} \exp \left\{-\omega_{n-1}^{1 /(n-1)}\left(\frac{2 K_{O} \omega_{n-1}}{(\log d / r)^{n-1}}\right)^{1 /(1-n)}\right\} \\
& \leq c_{n} \exp \left\{-\left(2 K_{O}\right)^{1 /(1-n)} \log d / r\right\} \leq C(n, K) r^{\alpha},
\end{aligned}
$$

where $\alpha=\left(2 K_{O}\right)^{1 /(1-n)}$. Finally since $r_{1} \approx\left|E^{*}\right|^{1 /(n-1)}=|E|^{1 /(n-1)}$ and since $E$ was arbitrary, we infer that

$$
\left|f^{-1}(\bar{B}(y, r) \cap \partial D)\right| \leq C(n, K) r^{\beta},
$$

where $\beta=(n-1)\left(2 K_{O}\right)^{1 /(1-n)}$.

To complete the proof, suppose that $F \subset \partial D$ is such that $\mathscr{H}_{\beta}(F)=$ 0 , where $\beta$ is as above. Fix $\varepsilon>0$ and cover $F$ by balls $B\left(y_{i}, r_{i}\right)$, $r_{i}<d / 2$, such that $\sum_{i} r_{i}^{\beta}<\varepsilon$. The preceding argument shows that

$$
\left|f^{-1}\left(\bar{B}\left(y_{i}, r_{i}\right) \cap \partial D\right)\right| \leq C(n, K) r_{i}^{\beta}
$$

for each $i$; in particular,

$$
\left|f^{-1} F\right| \leq\left|\bigcup_{i} f^{-1}\left(\bar{B}\left(y_{i}, r_{i}\right) \cap \partial D\right)\right| \leq C \sum_{i} r_{i}^{\beta}<C \varepsilon,
$$

where $C=C(n, K)$. Letting $\varepsilon \rightarrow 0$ yields $\left|f^{-1} F\right|=0$, as desired. The proof of Theorem A is complete. 
4. Proofs of Theorems $B$ and $B^{\prime}$. We begin with a lemma.

4.1. Lemma. Suppose that $D \subset \mathbf{R}^{n}$ is a domain such that $\partial D=$ $\partial D^{*}$, where $D^{*}=\mathbf{R}^{n} \backslash \bar{D}$ is an unbounded b-uniform domain, and let $y_{0} \in D$. Then there exists $\varepsilon=\varepsilon(n, b)>0$ such that

$$
\bmod \Gamma_{r, x_{0}} \leq \frac{(1-\varepsilon) \omega_{n-1}}{(\log d / r)^{n-1}}
$$

for $0<r<d=\operatorname{dist}\left(y_{0}, \partial D\right) / 2$ and $x_{0} \in \partial D$, where

$$
\Gamma_{r, x_{0}}=\Delta\left(\partial B\left(x_{0}, r\right) \cap D, \partial B\left(x_{0}, d\right) \cap D ; D\right) .
$$

Proof. Fix $x_{0} \in \partial D, 0<r<d$, and define

$$
\rho(x)=\left|x-x_{0}\right|^{-1}(\log d / r)^{-1}
$$

if $x \in\left(B\left(x_{0}, d\right) \cap D\right) \backslash B\left(x_{0}, r\right)$ and $\rho(x)=0$ otherwise. Then

$$
\bmod \Gamma_{r, x_{0}} \leq \int_{\mathbf{R}^{n}} \rho^{n} d x .
$$

Because $D^{*}$ is $b$-uniform, for each $r<t<d$ there is a point $z \in$ $\partial B\left(x_{0}, t\right)$ such that $B(z, t / 2 b) \subset D^{*}$. Hence a simple calculation verifies that

$$
\int_{\mathbf{R}^{n}} \rho^{n} d x \leq \frac{(1-\varepsilon) \omega_{n-1}}{(\log d / r)^{n-1}}
$$

with $\varepsilon=\varepsilon(b, n)>0$ as desired.

To prove Theorem $\mathrm{B}^{\prime}$, we first observe that because $D$ is uniform, $f$ extends to a quasimöbius embedding of $\partial \mathbf{B}^{n}$ into $\overline{\mathbf{R}}^{n}=\mathbf{R}^{n} \cup\{\infty\}$ by [V4, 5.6], and hence $D^{*}=\mathbf{R}^{n} \backslash \bar{D}$ is an unbounded $b^{\prime}$-uniform domain for some $b^{\prime}=b^{\prime}(n, K, b)$ by [V5, 5.10]. Then we apply the argument of [AK, Theorem 2.3] together with the previous lemma (see also [NP, Theorem 7]) to conclude that

$$
1-\left|f^{-1}(x)\right| \leq C \operatorname{dist}(x, \partial D)^{\alpha}
$$

holds for all $x \in D$, where $\alpha$ is as described in the theorem. The reasoning here is so similar to that in $[\mathbf{A K}]$, that the details are best left to the reader. Now condition (4.2) guarantees that $f^{-1}$ is $\alpha$-Hölder continuous in $D$ (see [GM, 2.24, 3.4]), and the theorem follows.

Finally, Theorem B is an immediate consequence of Theorem $\mathrm{B}^{\prime}$, as $f^{-1}$ extends to an $\alpha$-Hölder continuous mapping of $\partial D$.

5. Proof of Theorem C. We first establish the following lemma and indicate how Theorem $\mathrm{A}$ follows from Theorem $\mathrm{C}$ for $n \neq 4$. 
5.1. LEMMA. Let $f$ be a quasiconformal mapping from $\mathbf{B}^{n}$ into $\mathbf{R}^{n}$ and suppose that $n \neq 4$. Then there is a quasiconformal mapping $g$ from $\mathbf{B}^{n}$ into $\mathbf{R}^{n}$ such that $g=f$ on $\partial \mathbf{B}^{n}$ and $|D g|^{-r} \in L^{1}\left(\mathbf{B}^{n}\right)$ for any

$$
0<r<\frac{1}{\left(2 K_{O}(f)\right)^{1 /(n-1)}-1} .
$$

Proof. By [TV, Corollary 7.12] there is, for any $\varepsilon>0$, a quasiconformal mapping $g$ from $\mathbf{B}^{n}$ onto $D=f\left(\mathbf{B}^{n}\right)$ such that $k_{D}(f(x), g(x))$ $<\varepsilon$ and

$$
L^{-1} k_{\mathbf{B}^{n}}(x, y) \leq k_{D}(g(x), g(y)) \leq L k_{\mathbf{B}^{n}}(x, y)
$$

for all $x, y \in \mathbf{B}^{n}$; here $k_{*}$ denotes the quasihyperbolic metric in a domain. Standard estimates for the quasihyperbolic metric give

$$
\log \frac{|f(x)-g(x)|+d}{d} \leq k_{D}(f(x), g(x))<\varepsilon,
$$

where $d=\min \{\operatorname{dist}(f(x), \partial D), \operatorname{dist}(g(x), \partial D)\}$. Thus

$$
|f(x)-g(x)| \leq\left(e^{\varepsilon}-1\right) d<d / 2
$$

for $\varepsilon>0$ small enough. This implies

$$
\operatorname{dist}(g(x), \partial D) \geq \operatorname{dist}(f(x), \partial D) / 2 .
$$

On the other hand, the reader can verify that the proof of [AK, Theorem 2.3] gives

$$
\operatorname{dist}(f(x), \partial D) \geq C^{-1}(1-|x|)^{\left(2 K_{o}(f)\right)^{1 /(n-1)}}
$$

for some $C \geq 1$ independent of $x$, so that

$$
\operatorname{dist}(g(x), \partial D) \geq C^{-1}(1-|x|)^{\left(2 K_{o}(f)\right)^{1 /(n-1)}} .
$$

Because (5.2) guarantees that $g$ is locally bilipschitz satisfying

$$
|D g(x)| \geq C^{-1} \frac{\operatorname{dist}(g(x), \partial D)}{1-|x|}
$$

for almost every $x \in \mathbf{B}^{n}$, we arrive at the desired conclusion by combining this with (5.3) and integrating.

Suppose now that $f$ is as in Theorem A. In Theorem C take $s=$ $n-1$; then

$$
\frac{s r}{n-s+r}=\frac{(n-1) r}{r+1}
$$


which equals $(n-1)\left(2 K_{O}(f)\right)^{1 /(1-n)}$ if

$$
r=\frac{1}{\left(2 K_{O}(f)\right)^{1 /(n-1)}-1} .
$$

We thus can conclude Theorem $\mathrm{A}$ for $n \neq 4$ from Theorem $\mathrm{C}$ and Lemma 5.1 .

Before the proof of Theorem $\mathrm{C}$, we recall some facts about $p$ modulus and Hausdorff measure.

For the definition of $M_{p}(\Gamma)$, the p-modulus of a path family $\Gamma$, $1<p<\infty$, we refer to [V1, Chapter 1]. Notice in particular that $M_{n}(\Gamma)=\bmod \Gamma$. If $A$ is a set in $\mathbf{R}^{n}$, we say that $A$ has zero $p$ modulus if

$$
M_{p}\left(\Delta\left(A^{\prime}, \partial B ; B\right)\right)=0
$$

for each compact $A^{\prime} \subset A$ and for some (each) ball $B$ containing $A^{\prime}$. Next we suppose that $1<p \leq n$, for if $p>n$, no nonempty subset of $\mathbf{R}^{n}$ has zero $p$-modulus. Then we have

$$
M_{p}(A)=0 \Rightarrow \operatorname{dim}_{H}(A) \leq n-p
$$

and

$$
\mathscr{H}_{n-p}(A)<\infty \Rightarrow M_{p}(A)=0,
$$

whenever $A \subset \mathbf{R}^{n}$. See e.g. [V2].

To begin the proof, fix $0<s \leq n-1$ and let $E \subset \partial \mathbf{B}^{n}$ be a set such that $0<\mathscr{H}_{s}(E)<\infty$. We may assume that $E$ is compact, that $f$ has radial limits everywhere in $E$, and that those limits lie in a fixed ball.

Let $1<q<p \leq n$. We are going to estimate the $q$-modulus of the path family $\Gamma=\Delta\left(\bar{B}_{1 / 2}, E ; \mathbf{B}^{n}\right)$. Because $f$ is $\mathrm{ACL}_{q}$ in the sense of [V1, 26.5], we may assume by Fuglede's theorem [V1, 28.2] that $f$ is absolutely continuous on each path in $\Gamma$. Thus if $\rho$ is admissible for the image family $\Gamma^{\prime}=\Delta\left(f\left(\bar{B}_{1 / 2}\right), f(E) ; D\right)$, the function $\tilde{\rho}(x)=\rho(f(x))\left|f^{\prime}(x)\right|$ is admissible for $\Gamma$. Therefore, we have the following $q$-modulus estimate

$$
\begin{aligned}
& M_{q}(\Gamma) \leq \int_{\mathbf{B}^{n}} \rho^{q}(f(x))\left|f^{\prime}(x)\right|^{q} d x \\
& \quad \leq\left(\int_{\mathbf{B}^{n}} \rho^{p}(f(x))\left|f^{\prime}(x)\right|^{n} d x\right)^{q / p}\left(\int_{\mathbf{B}^{n}}\left|f^{\prime}(x)\right|^{q(p-n) /(p-q)} d x\right)^{(p-q) / p}
\end{aligned}
$$

by Hölder's inequality. The last integral converges if

$$
p \geq q \frac{n+r}{q+r} .
$$


Consequently, for these values of $p$ and $q$,

$$
M_{q}(\Gamma)^{p / q} \leq C \int_{\mathbf{B}^{n}} \rho^{p}(f(x))\left|f^{\prime}(x)\right|^{n} d x \leq C \int_{D} \rho(y)^{p} d y,
$$

where the quasiconformality of $f$ was used in the last inequality. Since $\rho$ was arbitrary,

$$
M_{q}(\Gamma)^{p / q} \leq C M_{p}\left(\Delta\left(f\left(\bar{B}_{1 / 2}\right), f(E) ; \mathbf{R}^{n}\right)\right) .
$$

On the other hand, by using the theory of $Q E D_{p}$-domains [K1] one can show that

$$
M_{q}(\Delta(E, \partial B(0,2) ; B(0,2))) \leq C(q, n) M_{q}(\Gamma),
$$

and hence

$$
M_{q}(E)>0 \Rightarrow M_{p}\left(\Delta\left(f\left(\bar{B}_{1 / 2}\right), f(E) ; \mathbf{R}^{n}\right)\right)>0,
$$

by (5.5). Finally, by again invoking the $Q E D_{p}$-theory [K1], it is not difficult to see that $M_{p}(f(E))>0$ if $M_{p}\left(\Delta\left(f\left(\bar{B}_{1 / 2}\right), f(E) ; \mathbf{R}^{n}\right)\right)>0$. We conclude that

$$
M_{q}(E)>0 \Rightarrow M_{p}(f(E))>0
$$

for all $1<q<p<n$ such that (5.4) holds. Now the theorem follows from the indicated relationship between modulus and Hausdorff measure. More precisely, choose $q=n-s$ and observe that $\operatorname{dim}_{H}(f E) \geq n-p$ for all $p$ satisfying (5.4).

6. Proof of Theorem D. We assume throughout this section that $f$ is a $K$-quasiconformal mapping of $\mathbf{B}^{n}$ onto a domain $D$. We recall two basic distortion properties.

If $x, y$ and $z$ belong to a hyperbolic ball of radius $R>0$ in $\mathbf{B}^{n}$, then

$$
|x-y| \leq t|x-z| \Rightarrow|f(x)-f(y)| \leq \eta(t)|f(x)-f(z)|
$$

for an increasing homeomorphism $\eta:[0, \infty) \rightarrow[0, \infty)$ that depends only on $n, K$, and $R$; see [V3, Theorem 2.4]. This property will be called the local quasisymmetry of $f$.

Similarly, if $x$ and $y$ lie in a hyperbolic ball with radius $R$, we have

$$
C^{-1} a_{f}(x) \leq a_{f}(y) \leq C a_{f}(x),
$$

where $C$ depends only on $n, K$, and $R$. This follows e.g. from the local quasisymmetry combined with the Koebe type result

$$
C^{-1} a_{f}(x) \leq \frac{\operatorname{dist}(f(x), \partial D)}{1-|x|} \leq C a_{f}(x),
$$


where $C$ depends only on $n$ and $K$ (see [AG1, Theorem 1.8]).

We start with two lemmata, similar to those in [P2]. The first was proved in [K2, Lemma 2.6].

6.3. LEMMA. If $\gamma \subset \mathbf{B}^{n}$ is a rectifiable curve with $\operatorname{diam} \gamma \geq$ $\operatorname{dist}\left(\gamma, \partial \mathbf{B}^{n}\right)$, then

$$
\operatorname{diam} f(\gamma) \leq C \int_{\gamma} a_{f}(x)|d x|
$$

where $C=C(n, K)$.

6.4. LemmA. Let $A \subset \partial \mathbf{B}^{n}$. If $a_{f}(r w) \leq M$ for all $0<r<1$ and $w \in A$, then

$$
\left|f\left(w_{1}\right)-f\left(w_{2}\right)\right| \leq C M\left|w_{1}-w_{2}\right|
$$

for all $w_{1}, w_{2} \in A$, where $C=C(n, K)$.

Proof. We observe first that the assumption and Lemma 6.3 guarantee that $f(w)$ exists as a radial limit for each $w \in A$, so the assertion makes sense. Next, we may suppose that $\left|w_{1}-w_{2}\right|<1 / 10$, for otherwise Lemma 6.3 implies

$$
\left|f\left(w_{1}\right)-f\left(w_{2}\right)\right| \leq \operatorname{diam} f A \leq C M \leq 10 C M\left|w_{1}-w_{2}\right| .
$$

Fix $0<r<1$ such that

$$
\left|r w_{1}-r w_{2}\right| \approx(1-r) \approx\left|w_{1}-w_{2}\right|
$$

Then

$$
\begin{aligned}
\left|f\left(w_{1}\right)-f\left(w_{2}\right)\right| \leq & \left|f\left(w_{1}\right)-f\left(r w_{1}\right)\right| \\
& +\left|f\left(r w_{1}\right)-f\left(r w_{2}\right)\right|+\left|f\left(r w_{2}\right)-f\left(w_{2}\right)\right| .
\end{aligned}
$$

Because, by the local quasisymmetry and (6.2),

$$
\begin{aligned}
\left|f\left(r w_{1}\right)-f\left(r w_{2}\right)\right| & \approx \operatorname{dist}\left(f\left(r w_{1}\right), \partial D\right) \approx a_{f}\left(r w_{1}\right)(1-r) \\
& \leq M(1-r) \approx M\left|w_{1}-w_{2}\right|,
\end{aligned}
$$

it suffices to show, by symmetry, that

$$
\left|f\left(r w_{1}\right)-f\left(w_{1}\right)\right| \leq C M(1-r) .
$$

But this follows from Lemma 6.3, and the proof is thereby complete.

6.5. Dyadic decomposition of $\partial \mathbf{B}^{n}$. We require a decomposition of $\partial \mathbf{B}^{n}$ into sets that correspond to the dyadic intervals on the boundary of the unit disk (or the dyadic subcubes of $\mathbf{R}^{n}$ ). To be precise 
with this construction, let $\mathbf{Q}^{n-1}=[-1,1]^{n-1}$ be the closed unit cube in $\mathbf{R}^{n-1}$ and consider the radial stretching $F: \mathbf{Q}^{n-1} \rightarrow \overline{\mathbf{B}}^{n-1}$ defined by

$$
F(x)=\frac{x}{\left|v_{x}\right|}, \quad x \in \mathbf{Q}^{n-1} \backslash\{0\},
$$

where $v_{x}$ is the unique point on $\partial \mathbf{Q}^{n-1}$ where the ray $t x, t \geq 0$, meets $\partial \mathbf{Q}^{n-1}$. Also, we set $F(0)=0$. Then $F$ is an $L$-bilipschitz mapping with $L$ depending only on $n$. Next, let $\pi: \partial \mathbf{B}^{n} \backslash\left\{e_{n}\right\} \rightarrow$ $\mathbf{R}^{n-1}$ be the stereographic projection

$$
\pi(x)=e_{n}+\frac{2\left(x-e_{n}\right)}{\left|x-e_{n}\right|^{2}} .
$$

Then

$$
H=\pi^{-1} \circ F: \mathbf{Q}^{n-1} \rightarrow \partial \mathbf{B}_{-}^{n}=\partial \mathbf{B}^{n} \cap\left\{x \in \mathbf{R}^{n}: x_{n} \leq 0\right\}
$$

defines an $L$-bilipschitz mapping with $L=L(n)$.

By dyadic cubes on $\partial \mathbf{B}^{n}$ we mean the members of the family

$$
\mathscr{D}=\left\{H(Q), H^{*}(Q): Q \text { is a closed dyadic cube in } \mathbf{Q}^{n-1}\right\},
$$

where $H^{*}$ denotes $H$ followed by the reflection $\left(x_{1}, \ldots, x_{n-1}, x_{n}\right) \mapsto$ $\left(x_{1}, \ldots, x_{n-1},-x_{n}\right)$. The members of $\mathscr{D}$ are also denoted by $Q$. By construction, it is readily seen that they share the following properties of the ordinary dyadic cubes:

(i) for a.e. point $w$ on $\partial \mathbf{B}^{n}$ there is a unique descending chain of dyadic cubes converging to $w$;

(ii) $|Q| \approx\left|Q^{\prime}\right|$ if $Q$ and $Q^{\prime}$ are within one generation apart.

Next, if $Q \in \mathscr{D}$, we let $w_{Q} \in Q$ denote the center of $Q$ and it is, by definition, the image of the center of the corresponding cube under $H\left(\right.$ or $\left.H^{*}\right)$. Moreover, $z_{Q} \in \mathbf{B}^{n}$ is a tent-point of $Q$ if it satisfies

$$
1-\left|z_{Q}\right| \approx\left|z_{Q}-w_{Q}\right| \approx \operatorname{diam} Q \approx|Q|^{1 /(n-1)}
$$

for some dimensional constants. Also observe that for each point $x \in \mathbf{B}^{n}$ there is $Q_{x} \in \mathscr{D}$ such that

$$
1-|x| \approx \operatorname{dist}\left(x, Q_{x}\right) \approx \operatorname{diam} Q_{x}
$$

for some dimensional constants.

From now on, we assume that a fixed choice of the point $z_{Q}$ has been made for each $Q \in \mathscr{D}$. 
6.6. Lemma. Let $Q \in \mathscr{D}$. Then there is $A \subset Q$ and $\delta=\delta(n)>0$ such that $|A| \geq \delta|Q|$ and that

$$
\left|f\left(z_{Q}\right)-f(w)\right| \leq M(n, K) \operatorname{dist}\left(f\left(z_{Q}\right), \partial D\right)
$$

for each $w \in A$.

Proof. We normalize $f\left(z_{Q}\right)=0$ and $\operatorname{dist}(0, \partial D)=1$. Fix $t>1$ and let $A_{t} \subset Q$ be the set of all points $w$ in $Q$ such that $|f(w)|>$ $t$. Consider the mapping $g=f \circ U_{Q}$, where $U_{Q}$ is a Möbius self mapping of the unit ball that carries 0 to $z_{Q}$. Then $g$ is a quasiconformal mapping with $K(g)=K(f)=K$, and $g(0)=0$. Let $\widetilde{A_{t}}=U_{Q}^{-1}\left(A_{t}\right), \widetilde{Q}=U_{Q}^{-1}(Q)$, and let $\Gamma_{t}$ denote the family of all radial segments joining $\bar{B}_{1 / 2}$ to $\widetilde{A}_{t}$. We have

$$
\bmod \Gamma_{t} \geq(\log 2)^{1-n}\left|\widetilde{A_{t}}\right|
$$

and hence

$$
\bmod g \Gamma_{t} \geq(\log 2)^{1-n}\left|\widetilde{A_{t}}\right| / K .
$$

The local quasisymmetry together with our normalization implies that $g\left(\bar{B}_{1 / 2}\right) \subset B(0, H)$ for some $H=H(n, K)$. Thus

$$
\bmod g \Gamma_{t} \leq \frac{\omega_{n-1}}{(\log t / H)^{n-1}}
$$

for $t>H$ so that

$$
\left|\widetilde{A}_{t}\right| \leq C(n, K)(\log t / H)^{1-n} \equiv \varphi(t),
$$

and since $|\widetilde{Q}| \approx 1$, we can choose $t=t(n, K)$ so large that

$$
\left|\widetilde{Q} \backslash \widetilde{A_{t}}\right| \geq|\widetilde{Q}|-\varphi(t) \geq C(n)>0 .
$$

Moreover, for any $E \subset \partial \mathbf{B}^{n}$

$$
\left|U_{Q}(E)\right|=\int_{E} J_{U_{Q}}(w) d \mathscr{H}_{n-1}(w)=\int_{E}\left|D U_{Q}(w)\right|^{n-1} d \mathscr{H}_{n-1}(w)
$$

and because $\left|D U_{Q}\right| \approx \operatorname{diam} Q$ on $\widetilde{Q}$, we obtain

$$
\left|\widetilde{Q} \backslash \widetilde{A_{t}}\right| \approx \frac{\left|U_{Q}\left(\widetilde{Q} \backslash \widetilde{A_{t}}\right)\right|}{|Q|} .
$$

Finally, since for $w \in \widetilde{Q} \backslash \widetilde{A_{t}}$ we have $|g(w)| \leq t$, the assertion follows. 
Proof of Theorem D. Our proof closely resembles that of Pommerenke's in [P2]. First observe $\partial \mathbf{B}^{n} \backslash E_{\infty}=\widetilde{E}_{0} \cup \widetilde{E}_{1}$, where

$$
\widetilde{E}_{0}=\left\{w \in \partial \mathbf{B}^{n}: \liminf _{r \rightarrow 1} a_{f}(r w)=0\right\} \quad \text { and } \quad \widetilde{E}_{1}=\partial \mathbf{B}^{n} \backslash E_{\infty} \backslash \widetilde{E}_{0}
$$

Now

$$
\widetilde{E}_{1}=\bigcup_{k=1}^{\infty} A_{k}, \quad \text { where } A_{k}=\left\{w \in \widetilde{E}_{1}: \limsup _{r \rightarrow 1} a_{f}(r w)<k\right\} .
$$

By Lemma $6.4 f$ is Lipschitz on $A_{k}$ so that $\left|f\left(A_{k}\right)\right|<\infty$; in particular, $f\left(\widetilde{E}_{1}\right)$ has $\sigma$-finite $\mathscr{H}_{n-1}$-measure and $f$ is absolutely continuous in $\widetilde{E}_{1}$.

We next show that $\widetilde{E}_{0}=E_{0} \cup A_{0}$, where $\left|E_{0}\right|=0$ and $\left|f\left(A_{0}\right)\right|=0$. Then we can set $E_{1}=\widetilde{E}_{1} \cup A_{0}$.

To this end, for each $w \in \widetilde{E}_{0}$ and $j=1,2, \ldots$ there is $r_{j, w}, 1-$ $1 / j<r_{j, w}<1$, such that

$$
a_{f}\left(r_{j, w} w\right)<2^{-j} .
$$

Let $Q_{j, w}$ be the largest dyadic cube in $\mathscr{D}$ containing $w$ with diam $Q_{j, w} \leq 1-r_{j, w}$; such a cube exists for almost every $w \in \partial \mathbf{B}^{n}$. Then the collection

$$
\mathscr{D}_{k}=\left\{Q_{j, w}: w \in \widetilde{E}_{0}, j \geq k\right\}
$$

covers almost all of $\widetilde{E}_{0}$, and by the Vitali covering theorem we can select a countable collection of essentially disjoint cubes $\left\{Q_{k, l}\right\} \subset \mathscr{D}_{k}$ such that

$$
\left|\widetilde{E}_{0} \cap\left(\bigcup Q_{k, l}\right)\right|=\left|\widetilde{E}_{0}\right|
$$

Next, Lemma 6.6 implies the existence of sets $A_{k, l} \subset Q_{k, l}$ and $\delta>0$ such that

$$
\left|A_{k, l}\right| \geq \delta\left|Q_{k, l}\right|
$$

and that

$$
f\left(A_{k, l}\right) \subset B\left(f\left(z_{k, l}\right), M \operatorname{dist}\left(f\left(z_{k, l}\right), \partial D\right)\right),
$$

where $z_{k, l}=z_{Q_{k, l}}$ and $M=M(n, K)$. We define

$$
E_{k}=\widetilde{E}_{0} \cap\left(\bigcup Q_{k, l}\right), \quad F_{k}=E_{k} \cap\left(\bigcup A_{k, l}\right),
$$

and

$$
A_{0}=\bigcap_{i=1}^{\infty} \bigcup_{k=i}^{\infty} F_{k}
$$


Next, we show that $\left|f\left(A_{0}\right)\right|=0$. It follows from (6.10) that each $f\left(F_{k}\right)$ can be covered by balls

$$
B\left(f\left(z_{k, l}\right), M \operatorname{dist}\left(f\left(z_{k, l}\right), \partial D\right)\right) \equiv B_{k, l} .
$$

Since the hyperbolic distance between $z_{k, l}$ and $r_{j, w} w$ (where $z_{k, l}$ corresponds to $Q_{k, l}=Q_{j, w}$ for some $j \geq k$ and $w \in \widetilde{E}_{0}$ ) is bounded by a constant $C=C(n)$, we have by (6.1), (6.2), and (6.8) that

$$
\begin{aligned}
\operatorname{diam} B_{k, l} & =2 M \operatorname{dist}\left(f\left(z_{k, l}\right), \partial D\right) \\
& \leq C\left(1-\left|z_{k, l}\right|\right) a_{f}\left(z_{k, l}\right) \leq C \operatorname{diam} Q_{k, l} 2^{-k} .
\end{aligned}
$$

Thus

$$
\sum_{l}\left(\operatorname{diam} B_{k, l}\right)^{n-1} \leq C 2^{-k(n-1)} \sum_{l}\left(\operatorname{diam} Q_{k, l}\right)^{n-1} \leq C 2^{-k(n-1)},
$$

where $C=C(n, K)$; here we used the fact that the cubes $Q_{k, l}$ are essentially disjoint. In consequence, since $f\left(A_{0}\right)$ belongs to $f\left(F_{i}\right) \cup$ $f\left(F_{i+1}\right) \cup \cdots$ for any $i=1,2, \ldots, f\left(A_{0}\right)$ can be covered by balls $B_{k, l}, k \geq i$, such that

$$
\sum_{k \geq i} \sum_{l}\left(\operatorname{diam} B_{k, l}\right)^{n-1} \leq C \sum_{k \geq i} 2^{-k(n-1)} \leq C 2^{-i(n-1)} .
$$

This implies $\left|f\left(A_{0}\right)\right|=0$.

It remains to show that $\left|E_{0}\right|=0$, where $E_{0}=\widetilde{E}_{0} \backslash A_{0}$. Now

$$
E_{0} \subset \bigcup_{i=1}^{\infty} \bigcap_{k=i}^{\infty} \bigcup_{l=1}^{\infty}\left(Q_{k, l} \backslash A_{k, l}\right) \cup E_{0,0},
$$

where $\left|E_{0,0}\right|=0$, and we want to show that $\left|G_{i}\right|=0$ for each $i=$ $1,2, \ldots$, where

$$
G_{i}=\bigcap_{k=i}^{\infty} \bigcup_{l=1}^{\infty}\left(Q_{k, l} \backslash A_{k, l}\right)
$$

Fix $i$. By construction, for almost every point $w$ in $G_{i}$ there is a descending chain of cubes of the form $Q_{k, l}, k \geq i$, that converge to $w$. It is then easy to see that $w$ cannot be a point of density of $G_{i}$ because

$$
\frac{\left|Q_{k, l} \cap G_{i}\right|}{\left|Q_{k, l}\right|} \leq \frac{\left|Q_{k, l} \backslash A_{k, l}\right|}{\left|Q_{k, l}\right|} \leq 1-\delta<1
$$

for all $k$ by (6.9). This completes the proof of Theorem $\mathrm{D}$.

7. Proofs of Theorems $\mathbf{E}$ and $\mathbf{F}$. Suppose that $f$ is as in Theorem $\mathbf{E}$. We can factor $f$ as $f=\varphi \circ h$, where $h: \mathbf{B}^{2} \rightarrow \mathbf{B}^{2}$ is a quasiconformal 
mapping with $h(0)=0$ and $\varphi: \mathbf{B}^{2} \rightarrow f\left(\mathbf{B}^{2}\right)$ is conformal. An easy argument shows that the complex dilatation $\mu_{h}$ of $h$ satisfies the Carleson measure condition of Theorem E, i.e. $\left|\mu_{h}(z)\right|^{2} /(1-|z|)$ is a Carleson measure in $\mathbf{B}^{2}$. Then [FKP, Theorem 3.3] shows that the boundary correspondence of $h$ induces an $A_{\infty}$ measure on the boundary of $\mathbf{B}^{2}$. To be more precise, the quasiconformal mapping $h$ gives rise to an elliptic operator

$$
L=-\operatorname{div} A(x) \nabla
$$

in the disk, where $A(x)=J_{h}(x)\left[D h(x)^{-1}\right]\left[D h(x)^{-1}\right]^{t}$ for a.e. $x \in \mathbf{B}^{2}$. A straightforward calculation shows that the ratio of

$$
\sup _{x \in B_{z}}\|A(x)-I d\|, \quad B_{z}=B(z,(1-|z|) / 2),
$$

and $\left|\mu_{h}(z)\right|$ is bounded from above and below by a constant independent of $z \in \mathbf{B}^{2}$; because the associated elliptic measure $\omega_{L}$ satisfies

$$
\omega_{L}^{0}(E)=\frac{|h(E)|}{2 \pi}
$$

for $E \subset \partial \mathbf{B}^{2}$ (see e.g. [GLM] or [HM]), the assertion follows from [FKP, 3.3].

In particular, we have $|A|=0$ if and only if $|h(A)|=0$ for $A \subset$ $\partial \mathbf{B}^{2}$. Makarov's theorem guarantees that there is a set $E^{\prime} \subset \partial \mathbf{B}^{2}$ such that $\left|E^{\prime}\right|=2 \pi$ and the Hausdorff dimension of $\varphi\left(E^{\prime}\right)$ equals 1 . Then $h^{-1}\left(E^{\prime}\right)=E$ is the desired set.

Next we prove Theorem F. Suppose that $f$ is as in Theorem $\mathrm{F}$ and let $E \subset \partial \mathbf{B}^{n}$ be such that $\operatorname{dim}_{H} E=s, 0<s \leq n-1$. The proof will show that there is no loss of generality in assuming that $f$ is bounded. Fix $\varepsilon>0$. For $w \in \partial \mathbf{B}^{n}$ and $r>0$ the domain

$$
D_{w, r}=B(w, r) \cap \mathbf{B}^{n}
$$

is $b$-uniform with $b$ independent of $w$ and $r$. By [AH, Theorem 1.3] there is $K_{1}=K_{1}(n, b)>1$ such that every bounded $K$ quasiconformal mapping $g$ of $D_{w, r}$ with $K \leq K_{1}$ can be extended to a $K_{2}$-quasiconformal mapping of $\mathbf{R}^{n}$; moreover, $K_{2} \rightarrow 1$ as $K \rightarrow 1$. Thus for fixed $\varepsilon>0$ we can choose $r>0$ so small that $f_{w, r} \equiv f \mid D_{w, r}$ extends to a $K_{2}$-quasiconformal mapping $\tilde{f}_{w, r}: \mathbf{R}^{n} \rightarrow \mathbf{R}^{n}$ with $s-\varepsilon \leq$ $s K_{2}^{1 /(1-n)} \leq s K_{2}^{1 /(n-1)} \leq s+\varepsilon$. Because $K$-quasiconformal mappings of $\mathbf{R}^{n}$ are Hölder continuous with exponent $K^{1 /(1-n)}$ on compact subsets, $f$ satisfies

$$
M_{w}^{-1}|x-y|^{1 / \alpha} \leq|f(x)-f(y)| \leq M_{w}|x-y|^{\alpha}, \quad \alpha=K_{2}^{1 /(1-n)},
$$


for all $x, y \in \bar{D}_{w, r}$ and for any $w \in \partial \mathbf{B}^{n}$. In particular, the Hausdorff dimension of $f\left(E \cap \bar{D}_{w, r}\right)$ is at most $s+\varepsilon$ and at least $s-\varepsilon$ by the choice of $r$. Finally, since $\partial \mathbf{B}^{n}$ can be covered by finitely many sets $\bar{D}_{w_{1}, r}, \ldots, \bar{D}_{w_{k}, r}$ we conclude that

$$
s-\varepsilon \leq \operatorname{dim}_{H} f(E) \leq s+\varepsilon,
$$

and the theorem follows by letting $\varepsilon \rightarrow 0$.

8. Open problems. In the following, $f$ is a $K$-quasiconformal mapping of $\mathbf{B}^{n}$ into $\mathbf{R}^{n}$.

Problem 1. Denote by $\mu(n, K)$ the supremum of all numbers $\alpha$ such that $\operatorname{dim}_{H} f(E) \geq \alpha$ whenever $E \subset \partial \mathbf{B}^{n}$ has positive $\mathscr{H}_{n-1}$ measure. By Theorem A, $\mu(n, K) \geq(n-1)(2 K)^{1 /(1-n)}$. Find a better lower bound for $\mu(n, K)$.

Problem 2. Similarly, define $\mu(n, K, s), 0<s \leq n-1$, to be the supremum of all numbers $\alpha$ such that $\operatorname{dim}_{H} f(E) \geq \alpha$ whenever $E \subset \partial \mathbf{B}^{n}$ has positive $\mathscr{H}_{s}$-measure. By Theorem C, $\mu(n, K, s) \geq$ $s r /(n-s+r)$, where $r=r(n, K)>0$. Find a better lower bound for $\mu(n, K, s)$. This is related to the unknown value of $r(n, K)$.

Problem 3. Let $r(n, K)>0$ be the supremum of all $r>0$ such that $|D f|^{-r} \in L^{1}\left(\mathbf{B}^{n}\right)$. Show that

$$
r(n, K) \geq \frac{1}{\left(2 K_{O}(f)\right)^{1 /(n-1)}-1} .
$$

Note that the inequality $r(2,1)<1$ follows from elementary distortion theorems for univalent functions. Brennan [B] has shown that $r(2,1)>1$ (see also [P1]) and hence it would be natural to expect that

$$
r(n, K)>\frac{1}{\left(2 K_{O}(f)\right)^{1 /(n-1)}-1} .
$$

This latter inequality would then give a better bound for $\mu(n, K, s)$ in Problems 1 and 2.

Problem 4. Give conditions that guarantee that $f$ has the Makarov property. Improve the Carleson measure condition in Theorem E.

Problem 5. Let $n \geq 3$ and suppose that

$$
K(r)=K\left(f \mid \mathbf{B}^{n} \backslash \bar{B}_{r}\right) \rightarrow 1, \quad r \rightarrow 1 .
$$


Under what conditions on $K(r)$ does $f$ have the Makarov property? Is there a higher dimensional analog of the Carleson measure condition in Theorem E? Mattila and Vuorinen [MV] have studied a related question.

Problem 6. Let $n \geq 3$ and define $E_{\infty}$ as in Theorem D. Construct a quasiconformal mapping such that $\left|E_{\infty}\right|>0$. Is it possible that $E_{\infty}=\partial \mathbf{B}^{n}$ ?

Problem 7. Let $n \geq 3$ and suppose that $|\partial D|<\infty$, where $D=$ $f\left(\mathbf{B}^{n}\right)$. Is $f \mid \partial \mathbf{B}^{n}$ absolutely continuous? What about $f^{-1} \mid \partial D$ ? This question was asked by Baernstein and Manfredi [BM, p. 846]. Gehring [G2] showed that $f \mid \partial \mathbf{B}^{n}$ is absolutely continuous if $f$ has an extension to a global quasiconformal mapping; Väisälä [V3] proved the same result under the weaker assumption that $f(D)$ be uniform. Recently the first author showed that $f \mid \partial \mathbf{B}^{n}$ is absolutely continuous if $\partial D$ has an $(n-1)$-tangent plane $\mathscr{H}_{n-1}$ almost everywhere [He]. The absolute continuity of $f^{-1} \mid \partial D$ is open in all these cases.

\section{REFERENCES}

[A] K. Astala, Area distortion of quasiconformal mappings, Acta Math., to appear.

[AG1] K. Astala and F. W. Gehring, Quasiconformal analogues of theorems of Koebe and Hardy-Littlewood, Michigan Math. J., 32 (1985), 99-107.

[AG2] _ Injectivity, the BMO norm and the universal Teichmüller space, J. Analyse Math., 46 (1986), 16-57.

[AH] K. Astala and J. Heinonen, On quasiconformal rigidity in plane and space, Ann. Acad. Sci. Fenn. Ser. A I Math., 13 (1988), 81-92.

[AK] K. Astala and P. Koskela, Quasiconformal mappings and global integrability of the derivative, J. Analyse Math., 57 (1991), 203-220.

[BM] A. Baernstein and J. J. Manfredi, Topics in quasiconformal mappings, Topics in modern harmonic analysis, Proc. Seminar held in Torino and Milano II (1982), 849-862.

[B] J. E. Brennan, The integrability of the derivative in conformal mapping, J. London Math. Soc., 18 (1978), 261-272.

[C] L. Carleson, On the distortion of sets on a Jordan curve under conformal mapping, Duke Math. J., 40 (1973), 547-559.

[CM] L. Carleson and N. G. Makarov, Some results connected with Brennan's conjecture, preprint (1992).

[FKP] R. A. Fefferman, C. E. Kenig and J. Pipher, The theory of weights and the Dirichlet problem for elliptic equations, Annals of Math., 134 (1991), 65-124.

[G1] F. W. Gehring, The $L^{p}$-integrability of the partial derivatives of a quasiconformal mapping, Acta Math., 130 (1973), 265-277. 
[G2] __ Lower dimensional absolute continuity properties of quasiconformal mappings, Math. Proc. Cambridge Philos. Soc., 78 (1975), 81-93.

[GM] F. W. Gehring and O. Martio, Lipschitz classes and quasiconformal mappings, Ann. Acad. Sci. Fenn. Ser. A I Math., 10 (1985), 203-219.

[GLM] S. Granlund, P. Lindqvist and O. Martio, F-harmonic measure in space, Ann. Acad. Sci. Fenn. Ser. A I Math., 7 (1982), 233-247.

[Ha] B. Hanson, Quasiconformal analogues of a theorem of Smirnov, (preprint, 1991).

[He] J. Heinonen, The boundary absolute continuity of quasiconformal mappings, Amer. J. Math., (to appear).

[HM] J. Heinonen and O. Martio, Estimates for F-harmonic measures and Øksendal's theorem for quasiconformal mappings, Indiana Univ. Math. J., 36 (1987), 659-683.

[K1] P. Koskela, Capacity extension domains, Ann. Acad. Sci. Fenn. Ser. A I Math. Dissertationes, 73 (1990).

[K2] - An inverse Sobolev lemma, Rev. Mat. Iberoamericana, (to appear).

[M1] N. G. Makarov, On the distortion of boundary sets under conformal mappings, Proc. London Math. Soc., 51 (1985), 369-384.

[M2] _ Conformal mapping and Hausdorff measures, Ark. Math., 25 (1987), 41-89.

[M3] _ Probability methods in the theory of conformal mapping (in Russian), Algebra and Analysis, 1 (1989), 1-59.

[MV] P. Mattila and M. Vuorinen, Linear approximation property, Minkowski dimension, and quasiconformal spheres, J. London Math. Soc., 42 (1990), 249266.

[N] R. Näkki, Prime ends and quasiconformal mappings, J. Analyse Math., 35 (1979), 13-40.

[NP] R. Näkki and B. P. Palka, Lipschitz conditions and quasiconformal mappings, Indiana Univ. Math. J., 31 (1982), 377-401.

[P1] Ch. Pommerenke, On the integral means of the derivative of a univalent function, J. London Math. Soc., 32 (1985), 254-258.

[P2] _ On conformal mapping and linear measure, J. Analyse Math., 46 (1986), 231-238.

[Re] Yu. G. Reshetnyak, Stability theorems in geometry and analysis (in Russian), Nauka, Novosibirsk, 1982.

[Ro] S. Rohde, On conformal welding and quasicircles, Michigan Math. J., 38 (1991), 111-116.

[S] J. Sarvas, Symmetrization of condensers in $n$-space, Ann. Acad. Sci. Fenn. Ser. A I Math., 522 (1972), 1-44.

[T1] P. Tukia, A quasiconformal group not isomorphic to a Möbius group, Ann. Acad. Sci. Fenn. Ser. A I Math., 6 (1981), 149-160.

[T2] _ Hausdorff dimension and quasisymmetric mappings, Math. Scand., 65 (1989), 152-160.

[TV] P. Tukia and J. Väisälä, Lipschitz and quasiconformal approximation and extension, Ann. Acad. Sci. Fenn. Ser. A I Math., 6 (1981), 303-342.

[V1] J. Väisälä, Lectures on n-Dimensional Quasiconformal Mappings, Lecture Notes in Math., vol. 229, Springer-Verlag, 1971.

[V2] _- Capacity and measure, Michigan Math. J., 22 (1975), 1-3.

[V3] Quasisymmetric embeddings in euclidean spaces, Trans. Amer. Math.

Soc., 264 (1981), 191-204.

[V4] _ Quasimöbius maps, J. Analyse Math., 44 (1984/85), 218-234. 
[V5] - Invariants for quasisymmetric, quasimöbius and bilipschitz maps, J. Analyse Math., 50 (1988), 201-223.

[V6] _ Quasiconformal maps and positive boundary measure, Analysis, 9 (1989), 205-216.

[Vu] M. Vuorinen, Conformal Geometry and Quasiregular Mappings, Lecture Notes in Math., vol. 1319, Springer-Verlag, New York, 1988.

Received September 15, 1992 and in revised form January 26, 1993. The first author was supported by NSF Grant DMS 91-48183 and The Academy of Finland.

UNIVERSITY OF MiCHIGAN

ANN ARBOR, MI 48109

AND

UNIVERSITY OF JYVÄSKYLÄ

SF-40100 JYVÄSKYLÄ, FINLAND 


\section{PACIFIC JOURNAL OF MATHEMATICS}

Founded by

E. F. BECKENBACH (1906-1982) F. Wolf (1904-1989)

\section{EDITORS}

Sun-Yung A. Chang

(Managing Editor)

University of California

Los Angeles, CA 90024-1555

chang@math.ucla.edu

\section{F. Michael Christ}

University of California

Los Angeles, CA 90024-1555

christ@math.ucla.edu

Herbert Clemens

University of Utah

Salt Lake City, UT 84112

clemens@math.utah.edu

\author{
ThOMAs ENRIGHT \\ University of California, San Diego \\ La Jolla, CA 92093 \\ tenright@ucsd.edu \\ Nicholas ERcolaNI \\ University of Arizona \\ Tucson, AZ 85721 \\ ercolani@math.arizona.edu \\ R. FINN \\ Stanford University \\ Stanford, CA 94305 \\ finn@gauss.stanford.edu \\ VAUGHAN F. R. Jones \\ University of California \\ Berkeley, CA 94720 \\ vfr@math.berkeley.edu
}

\author{
STEVEn KerCKHOFF \\ Stanford University \\ Stanford, CA 94305 \\ spk@gauss.stanford.edu
}

Martin ScharlemanN

University of California

Santa Barbara, CA 93106

mgscharl@math.ucsb.edu

\author{
Harold Stark \\ University of California, San Diego \\ La Jolla, CA 92093 \\ V. S. VARADARAJAN \\ University of California \\ Los Angeles, CA 90024-1555 \\ vsv@math.ucla.edu
}

\section{SUPPORTING INSTITUTIONS}

UNIVERSITY OF ARIZONA

UNIVERSITY OF BRITISH COLUMBIA

CALIFORNIA INSTITUTE OF TECHNOLOGY

UNIVERSITY OF CALIFORNIA

UNIVERSITY OF MONTANA

UNIVERSITY OF NEVADA, RENO

NEW MEXICO STATE UNIVERSITY

OREGON STATE UNIVERSITY

\author{
UNIVERSITY OF OREGON \\ UNIVERSITY OF SOUTHERN CALIFORNIA \\ STANFORD UNIVERSITY \\ UNIVERSITY OF HAWAII \\ UNIVERSITY OF UTAH \\ WASHINGTON STATE UNIVERSITY \\ UNIVERSITY OF WASHINGTON
}

The Supporting Institutions listed above contribute to the cost of publication of this Journal, but they are not owners or publishers and have no responsibility for its content or policies.

Mathematical papers intended for publication in the Pacific Journal of Mathematics should be in typed form or offset-reproduced (not dittoed), double spaced with large margins. Please do not use built up fractions in the text of the manuscript. However, you may use them in the displayed equations. Underline Greek letters in red, German in green, and script in blue. The first paragraph must be capable of being used separately as a synopsis of the entire paper. In particular it should contain no bibliographic references. Please propose a heading for the odd numbered pages of less than 35 characters. Manuscripts, in triplicate, may be sent to any one of the editors. Please classify according to the 1991 Mathematics Subject Classification scheme which can be found in the December index volumes of Mathematical Reviews. Supply name and address of author to whom proofs should be sent. All other communications should be addressed to the managing editor, or Julie Honig, University of California, Los Angeles, California 90024-1555.

There are page-charges associated with articles appearing in the Pacific Journal of Mathematics. These charges are expected to be paid by the author's University, Government Agency or Company. If the author or authors do not have access to such Institutional support these charges are waived. Single authors will receive 75 free reprints; joint authors will receive a total of 100 free reprints. Additional copies may be obtained at cost in multiples of 50 .

The Pacific Journal of Mathematics (ISSN 0030-8730) is published monthly except for July and August. Regular subscription rate: $\$ 215.00$ a year (10 issues). Special rate: $\$ 108.00$ a year to individual members of supporting institutions.

Subscriptions, orders for numbers issued in the last three calendar years, and changes of address should be sent to Pacific Journal of Mathematics, P.O. Box 4163, Berkeley, CA 94704-0163, U.S.A. Old back numbers obtainable from Kraus Periodicals Co., Route 100, Millwood, NY 10546.

The Pacific Journal of Mathematics at University of California, c/o Department of Mathematics, 981 Evans Hall, Berkeley, CA 94720 (ISSN 0030-8730) is published monthly except for July and August. Second-class postage paid at Berkeley, CA 94704, and additional mailing offices. POSTMASTER: send address changes to Pacific Journal of Mathematics, P.O. Box 4163, Berkeley, CA 94704-0163.

PUBLISHED BY PACIFIC JOURNAL OF MATHEMATICS at University of California, Berkeley, CA 94720, A NON-PROFIT CORPORATION

This publication was typeset using $\mathcal{A} \mathcal{M} \mathcal{S}-\mathrm{T}_{\mathrm{E}} \mathrm{X}$,

the American Mathematical Society's $T_{E} X$ macro system.

Copyright (c) 1994 by Pacific Journal of Mathematics 


\section{PACIFIC JOURNAL OF MATHEMATICS}

Volume $165 \quad$ No. $1 \quad$ September 1994

The effect of dimension on certain geometric problems of irregularities of 1 distribution

RALPH ALEXANDER

The structure of $\mathrm{sl}(2,1)$-supersymmetry: irreducible representations and primitive ideals

Didier ARnAL, HÉdi BENAMOR and GEORges PinCZON

Periods and Lefschetz zeta functions

Josefina CASASAYas, JaUme Llibre and ANA Nunes

On closed hypersurfaces of constant scalar curvatures and mean curvatures in 67 $S^{n+1}$

\section{SHAOPING CHANG}

$R$-groups and elliptic representations for $\mathrm{SL}_{n}$

DAVID GOLDBERG

The boundary distortion of a quasiconformal mapping

JuHA HeINONEN and PEKKA KosKela

Strongly approximately transitive group actions, the Choquet-Deny theorem, 115 and polynomial growth

\section{WOJCIECH JAWORSKI}

$q$-canonical commutation relations and stability of the Cuntz algebra

PALle E. T. Jorgensen, L. M. Schmitt and Reinhard Frank

WERNER

Complete open manifolds of non-negative radial curvature

YOSHIROH MACHIGASHIRA

Perturbations of certain reflexive algebras

DAVID RYDER PITTS

Interpolation submanifolds of the unitary group 